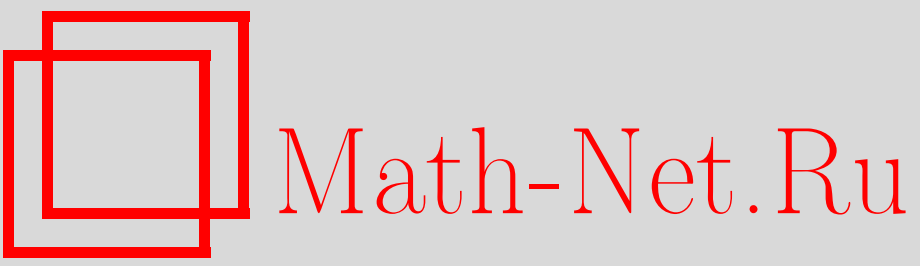

А. О. Смирнов, Об одном классе эллиптических решений уравнения Буссинеска, TMФ, 1996, том 109, номер 3, 347-356

DOI: https://doi.org/10.4213/tmf1232

Использование Общероссийского математического портала Math-Net.Ru подразумевает, что вы прочитали и согласны с пользовательским соглашением

http://www.mathnet.ru/rus/agreement

Параметры загрузки:

IP : 35.173 .219 .149

26 апреля 2023 г., 13:57:07 
ТЕОРЕТИЧЕСКАЯ

И МАТЕМАТИЧЕСКАЯ

ФИЗИКА

Том 109, № 3

декабрь, 1996

А. О. Смирнов*

\section{ОБ ОДНОМ КЛАССЕ ЭЛЛИПТИЧЕСКИХ РЕШЕНИЙ УРАВНЕНИЯ БУССИНЕСКА}

Рассмотрен анзац кривых Кричевера, ассоциированных с двоякопериодическими конечнозонными решениями уравнения Буссинеска, выраженными через эквиангармонические эллиптические ю-функции Вейерштрасса. Приведены примеры.

\section{ВВЕДЕНИЕ}

Эллиптические конечнозонные решения интегрируемых нелинейных уравнений представляют интерес не только сами по себе, как описывающие периодические (по времени и/или по координате) нелинейные процессы, они также могут быть использованы для изучения динамики интегрируемых систем частиц [1-3] или для исследований линейных обыкновенных дифференциальных или разностных уравнений, аналогичных уравнениям Ламе или Требиха-Вердье (см., например, [2-16]).

При исследовании условий периодичности были найдены эллиптические по различным переменным конечнозонные решения основных интегрируемых нелинейных уравнений [4-23]. Однако эти уравнения несмотря на все различия имеют одну общую черту - их конечнозонные решения строятся по гиперэллиптической кривой. Уравнениям, конечнозонные решения которых ассоциированы с негиперэллиптическими кривыми, в первую очередь это уравнения Кадомцева-Петвиашвили (КП) [2] и Буссинеска [24-26], уделено гораздо меньше внимания, несмотря на то что эллиптические по $x$ решения уравнения Буссинеска играют такую же большую роль при изучении уравнения Альфана и его аналогов [27, 28 (ур. 3.9-3.12), 15, 29-31], как и эллиптические решения уравнения Кортевега-де Фриза (КдФ) при исследовании оператора Шредингера с потенциалами Ламе и Требиха-Вердье.

В данной работе описывается класс кривых, ассоциированных с эллиптическими по $x$ решениями уравнения Буссинеска.

\section{1. КОНЕЧНОЗОННЫЕ РЕШЕНИЯ УРАВНЕНИЯ БУССИНЕСКА}

Следуя Кричеверу [32, 33], рассмотрим риманову поверхность (РП) Г рода $g$ с выделенной на ней точкой $\mathcal{P}_{\infty}$. Выберем на Г базис циклов $\gamma^{t}=\left(a_{1}, \ldots, a_{g}, b_{1}, \ldots, b_{g}\right)$ с матрицей пересечения

$$
C_{0}=\left(\begin{array}{cc}
0 & \mathrm{I} \\
-\mathrm{I} & 0
\end{array}\right)
$$

\footnotetext{
${ }^{*}$ Санкт-Петербургская государственная академия аэрокосмического приборостроения
} 
Ему соответствует нормированный базис голоморфных дифференциалов

$$
\oint_{a_{k}} d \mathcal{U}_{j}=\delta_{k j}, \quad k, j=1, \ldots, g .
$$

Хорошо известно, что матриша периодов

$$
B_{j k}=\oint_{b_{j}} d \mathcal{U}_{k}, \quad j, k=1, \ldots, g,
$$

есть симметрическая матрица с положительно определенной мнимой частью.

По матрице периодов $B$ построим тэта-функцию с характеристиками $\alpha, \beta \in \mathbb{R}^{g}$ :

$$
\begin{aligned}
\Theta[\alpha ; \beta](u \mid B) & \equiv \Theta[\alpha ; \beta](u)= \\
& =\sum_{m \in \mathbb{Z}^{g}} \exp \{\pi i\langle B(m+\alpha), m+\alpha\rangle+2 \pi i\langle m+\alpha, u+\beta\rangle\}, \\
\Theta[0 ; 0](u \mid B) & \equiv \Theta(u \mid B) \equiv \Theta(u),
\end{aligned}
$$

где $u \in \mathbb{C}^{g}$, суммирование ведется по целочисленной $g$-мерной решетке, а угловые скобки $\langle$,$\rangle обозначают евклидово скалярное произведение.$

Определим на $\Gamma$ нормированные абелевы интегралы второго рода $\Omega_{j}(P), P \in \Gamma$, с единственными полюсами в выделенной точке $\mathcal{P}_{\infty}$ :

$$
\begin{gathered}
\Omega_{j}(P)=\xi^{-j}+c_{j} \xi+O\left(\xi^{2}\right), \quad P \rightarrow \mathcal{P}_{\infty} \\
\oint_{a_{k}} d \Omega_{j}=0, \quad k=1, \ldots, g
\end{gathered}
$$

$\xi$ - локальный параметр в окрестности точки $\mathcal{P}_{\infty}$.

Пусть функция $\Psi\left(P, x, t_{1}, \ldots, t_{N}\right)$ удовлетворяет следуюшим условиям (аксиомам Бейкера-Ахиезера):

1) $\Psi\left(P, x, t_{1}, \ldots, t_{N}\right)$ есть однозначная функция от точки $P$ римановой поверхности $\Gamma$, мероморфная на $\Gamma$, за исключением точки $\mathcal{P}_{\infty}$, и имеюшая $g$ простых полюсов в точках $\mathcal{P}_{j}$, которые образуют дивизор обшего положения;

2) $\Psi(P, 0,0, \ldots, 0)=1$;

$3)$ в точке $\mathcal{P}_{\infty}$ функция $\Psi\left(P, x, t_{1}, \ldots, t_{N}\right)$ имеет сушественную особенность вида

$$
\begin{aligned}
\Psi\left(P, x, t_{1}, \ldots, t_{N}\right) \sim & \exp \left\{\xi^{-1} x+\sum_{i=1}^{N} \xi^{-i-1} t_{i}\right\} \times \\
& \times\left(1+\sum_{m=1}^{\infty} \psi_{m}\left(x, t_{1}, \ldots, t_{N}\right) \xi^{m}\right), \quad P \rightarrow \mathcal{P}_{\infty} .
\end{aligned}
$$

Тогда она этими условиями определяется однозначно и может быть построена по формуле

$$
\begin{aligned}
\Psi\left(P, x, t_{1}, \ldots, t_{N}\right)= & \exp \left\{\Phi\left(x, t_{1}, \ldots, t_{N}\right)\right\} \times \\
& \times \frac{\Theta\left(\mathcal{U}(P)-\mathcal{U}\left(\mathcal{P}_{\infty}\right)+\varphi\left(x, t_{1}, \ldots, t_{N}\right)+\Delta\right) \Theta(\Delta)}{\Theta\left(\mathcal{U}(P)-\mathcal{U}\left(\mathcal{P}_{\infty}\right)+\Delta\right) \Theta\left(\varphi\left(x, t_{1}, \ldots, t_{N}\right)+\Delta\right)},
\end{aligned}
$$


где

$$
\begin{gathered}
\Phi\left(x, t_{1}, \ldots, t_{N}\right)=\Omega_{1}(P) x+\sum_{i=1}^{N} \Omega_{i+1}(P) t_{i}, \\
\varphi\left(x, t_{1}, \ldots, t_{N}\right)=U x+\sum_{i=1}^{N} V_{i} t_{i}, \quad \Delta=\mathcal{U}\left(\mathcal{P}_{\infty}\right)-\mathcal{K}-\sum_{j=1}^{g} \mathcal{U}\left(\mathcal{P}_{j}\right),
\end{gathered}
$$

$2 \pi i U, 2 \pi i V_{i}$ - векторы $b$-периодов абелевых интегралов $\Omega_{1}(P), \Omega_{i+1}(P)$, соответственно; $\mathcal{K}$ - вектор римановых констант [33-35].

Tеорема $1[32,33]$. Функиия $\Psi\left(P, x, t_{1}, \ldots, t_{N}\right)$ является решением системы линейных дифференциальных уравнений

$$
\partial_{t_{j}} \psi=\partial_{x}^{j+1} \psi-\sum_{m=1}^{j} u_{j m}\left(x, t_{1}, \ldots, t_{N}\right) \partial_{x}^{j-m} \psi,
$$

где $u_{j m}\left(x, t_{1}, \ldots, t_{N}\right)$ - функции, зависящие от $x, t_{1}, \ldots, t_{N}$ и не зависящие от $P$.

В частности, если $N=3$, то (при $y \equiv t_{1}, t \equiv t_{2}, z \equiv t_{3}$ )

$$
\begin{aligned}
\partial_{y} \psi & =\partial_{x}^{2} \psi-u \psi \\
\partial_{t} \psi & =\partial_{x}^{3} \psi-\frac{3}{2} u \partial_{x} \psi-\left(\frac{3}{4} u_{x}+w\right) \psi, \\
\partial_{z} \psi=\partial_{x}^{4} \psi & -2 u \partial_{x}^{2} \psi-\left(2 u_{x}+\frac{4}{3} w\right) \partial_{x} \psi- \\
- & \left(\frac{5}{6} u_{x x}-\frac{1}{2} u^{2}+\frac{2}{3} w_{x}+v\right) \psi,
\end{aligned}
$$

где

$$
\begin{aligned}
u(x, y, t, z) & =-2 \partial_{x}^{2} \ln \Theta\left(U x+V_{1} y+V_{2} t+V_{3} z+\Delta\right)+2 c_{1}, \\
w(x, y, t, z) & =-\frac{3}{2} \partial_{x} \partial_{y} \ln \Theta\left(U x+V_{1} y+V_{2} t+V_{3} z+\Delta\right)+\frac{3}{2} c_{2}, \\
v(x, y, t, z) & =-\frac{4}{3} \partial_{x} \partial_{t} \ln \Theta\left(U x+V_{1} y+V_{2} t+V_{3} z+\Delta\right)+\frac{4}{3} c_{3} .
\end{aligned}
$$

СлЕДСТвИЕ $1[32,33]$. Условием совместности уравнений (1.3а) и (1.3б) является уравнение КП

$$
\left\{\begin{array}{l}
u_{y}=\frac{4}{3} w_{x} \\
u_{t}=w_{y}+\frac{1}{4} u_{x x x}-\frac{3}{2} u u_{x} .
\end{array}\right.
$$


Условиями совместности уравнений (1.3а), (1.3в) и (1.3б), (1.3в) являются "высшие" уравнения КП:

$$
\begin{aligned}
& \left\{\begin{array}{l}
u_{y}=\frac{4}{3} w_{x} \\
w_{y}=\frac{3}{2} v_{x}+\frac{3}{2} u u_{x}-\frac{1}{4} u_{x x x} \\
u_{z}=v_{y}+\frac{4}{9} w_{x x x}-\frac{4}{3} w u_{x}-\frac{4}{3} u w_{x}
\end{array}\right. \\
& \left\{\begin{aligned}
u_{t} & =\frac{3}{2} v_{x} \\
u_{z} & =\frac{8}{9} w_{t}+\frac{4}{9} w_{x x x}-\frac{4}{3} w u_{x}-\frac{4}{3} u w_{x}, \\
w_{z} & =v_{t}+\frac{1}{4} v_{x x x}-\frac{4}{3} w w_{x}+\frac{3}{2} u_{x} u_{x x}+ \\
& +\frac{3}{4} u u_{x x x}-\frac{3}{2} u^{2} u_{x}-\frac{1}{12} u_{x x x x x} .
\end{aligned}\right.
\end{aligned}
$$

ЗАмЕчАниЕ $1[33,35]$. Пусть $\Gamma$-гиперэллиптическая поверхность ипусть точка $\mathcal{P}_{\infty}$ является точкой ветвления этой поверхности. Тогда на Г существует мероморфная функция $E(P)$ с единственным полюсом второго порядка в точке $\mathcal{P}_{\infty}$. Если мы теперь выберем в качестве локального параметра в ее окрестности величину $\xi=[E(P)]^{-1 / 2}$, то в этом случае решение уравнения КП (1.5) не будет зависеть от $y$ :

$$
\Omega_{2}(P)=E(P), \quad V_{1}=0 .
$$

Иначе говоря, функция $u(x, t, z)(1.4 \mathrm{a})$ при любом значении параметра $z$ будет решением уравнения $\mathrm{K}$ д $\Phi$

$$
4 u_{t}-u_{x x x}+6 u u_{x}=0,
$$

а функция $\Psi(P, x, t, z)$ будет собственной функцией оператора Шредингера (см. (1.3a))

$$
\partial_{x}^{2} \psi-u \psi=E \psi .
$$

ЗАмечание $2[33,24-26]$. Если же на РП Г сушествует мероморфная функция $E(P)$ с единственным полюсом третьего порядка в точке $\mathcal{P}_{\infty}$, то, выбрав в качестве локального параметра в ее окрестности величину $\xi=[E(P)]^{-1 / 3}$, мы добьемся независимости решения уравнения КП от $t$ :

$$
\Omega_{3}(P)=E(P), \quad V_{2}=0 .
$$

При этом функция $u(x, y, z)$ при любом значении параметра $z$ будет решением уравнения Буссинеска

$$
3 u_{y y}+\left(u_{x x x}-6 u u_{x}\right)_{x}=0,
$$

а функция $\Psi(P, x, y, z)$ будет собственной функцией оператора третьего порядка:

$$
\partial_{x}^{3} \psi-\frac{3}{2} u \partial_{x} \psi-\left(\frac{3}{4} u_{x}+w\right) \psi=E \psi
$$

Это возможно только в двух случаях:

а) когда РП Г Гмеет род $g=2$ и точка $\mathcal{P}_{\infty}$ не является точкой Вейерштрасса [36-38];

б) когда РП Г негиперэллиптическая, а точка $\mathcal{P}_{\infty}$ является точкой Вейерштрасса, допускаюшей сушествование вьшшеупомянутой мероморфной функции $E(P)$. 


\section{2. КРИВЫЕ КРИЧЕВЕРА ДЛЯ УРАВНЕНИЯ БУССИНЕСКА. СЛУЧАЙ $g_{2}=0$}

Рассмотрим риманову поверхность $\Gamma=\{(k, \alpha)\}$ рода $g, n$-листно $(n \geqslant 2)$ накрываюшую эквиангармоническую эллиптическую кривую $\Gamma_{0}^{\prime}$ :

$$
\Gamma_{0}^{\prime}: \quad\left\{\wp^{\prime}(\alpha)\right\}^{2}=4 \wp^{3}(\alpha)-g_{3} \equiv 4 \prod_{j=1}^{3}\left(\wp(\alpha)-e_{j}\right),
$$

и удовлетворяюшую следуюшим требованиям:

1)

$$
\Gamma: \mathcal{R}(k, \alpha) \equiv k^{n}+\sum_{j=2}^{n} r_{j}(\alpha) k^{n-j}=0
$$

где $r_{j}(\alpha)$ - эллиптические функции от $\alpha \in \Gamma_{0}^{\prime}$ с полюсами в точках $\alpha=0$;

2 ) в окрестности $\alpha=0$ функция $\mathcal{R}(k, \alpha)$ представима в виде

$$
\mathcal{R}(k, \alpha)=\left(k-\frac{n-1}{\alpha}+b_{n}(\alpha)\right) \prod_{j=1}^{n-1}\left(k+\frac{1}{\alpha}+b_{j}(\alpha)\right) ;
$$

$3)$ на Г сушествует голоморфный автоморфизм третьего порядка $(\varepsilon=\exp \{2 \pi i / 3\})$ :

$$
\tau: \quad(k, \alpha) \rightarrow\left(\varepsilon^{2} k, \varepsilon \alpha\right),
$$

имеюший $g+2$ неподвижные точки.

ЗАмЕчаниЕ 3 . Из п. 3,2 следует, что выделенная точка $\mathcal{P}_{\infty}$ неподвижна относительно автоморфизма $\tau$, переставляюшего листы $\Gamma=\{(\chi, E)\}$ :

$$
\begin{aligned}
\chi^{3}= & \prod_{j=1}^{g+1}\left(E-E_{j}\right)^{m_{j}}, \quad \tau:(\chi, E) \rightarrow(\varepsilon \chi, E), \\
& m_{j} \in\{1 ; 2\}, \quad \sum_{j=1}^{g+1} m_{j} \not \equiv 0(\bmod 3),
\end{aligned}
$$

и что функция $E$ является мероморфной функцией с единственным полюсом третьего порядка в точке $\mathcal{P}_{\infty}=(\infty, \infty)$.

ЗАмечАниЕ 4. Используя формулу Римана-Гурвица [35, 12], нетрудно получить, что род $g$ поверхности $\Gamma ~(2.1)-(2.4)$ равен $p+1$, где $2 p$ - число нулей функции $\partial_{k} \mathcal{R}(k, \alpha)$, не лежаших "над" $\alpha=0$ и отличных от нулей функции $\partial_{\alpha} \mathcal{R}(k, \alpha)$ (с учетом кратности).

Tеорема 2. Решение и(x,y,z) уравнения Буссинеска (1.7), построенное по римановой поверхности $\Gamma_{g}^{n}(2.1)-(2.4)$ и отмеченной точке $\mathcal{P}_{\infty}$, является әллиптической функиией по $x$ :

$$
u(x, y, z)=2 \sum_{j=1}^{n} \wp\left(x-x_{j}(y, z)\right)+\text { const },
$$

где $\wp(x)$ - эллиптическая функиия Вейеритрасса с периодами накрываемой поверхности (2.1), $x_{j}(y, z)$ - нули тәта-функции $\Theta\left(U x+V_{1} y+V_{3} z+\Delta \mid B\right)$ как функиии от переменной $x$. 
ДокаЗАТЕЛЬСтво. Выберем в качестве мероморфной функции $E(P)$ функцию, инвариантную относительно автоморфизма $\tau(2.4)$ и имеюшую единственный полюс третьего порядка в точке $\mathcal{P}_{\infty}$ вида

$$
E(P)=-\frac{n^{3}}{\alpha^{3}}+\varkappa+O\left(\alpha^{3}\right) \equiv \xi^{-3}+O\left(\xi^{3}\right), \quad P \rightarrow \mathcal{P}_{\infty}
$$

Из (2.7) вытекает, что нормированный абелев дифференциал второго рода $d \Omega_{1}$ будет иметь следующую асимптотику в окрестности точки $\mathcal{P}_{\infty}$ :

$$
d \Omega_{1}=\left(-\frac{1}{\xi^{2}}+c_{1}+O(\xi)\right) d \xi=\left(\frac{n}{\alpha^{2}}-\frac{c_{1}}{n}+O(\alpha)\right) d \alpha
$$

Применяя теорему Лиувилля, нетрудно показать, что нормированные абелевы дифференциалы второго рода $d \Omega_{1}$ и

$$
d \Omega^{0}=(\wp(\alpha)+\eta / \omega) d \alpha
$$

определенные на Г и $\Gamma_{0}^{\prime}$, соответственно, связаны соотношением

$$
\sigma^{*} d \Omega^{0} \equiv d \Omega_{1}+d k
$$

с точностью до голоморфных дифференциалов. Здесь $\sigma$ - накрывающее отображение, $\sigma: \Gamma \rightarrow \Gamma_{0}^{\prime}, \sigma(k, \alpha)=\alpha ; 2 \omega, 2 \omega^{\prime}-$ периоды тора $(2.1) ; \eta=\zeta(\omega), \zeta(\alpha)$ - эллиптическая функция Вейерштрасса.

Теперь легко видеть, что утверждение теоремы 2 является следствием теоремы о редукции многомерной тэта-функции, накрывающей эллиптическую $[18,14,20,21]$, равенства (2.10) и формул связи эллиптических функций Вейерштрасса с одномерными тэта-функциями [39].

ЗАМЕЧАНИЕ 5. Из теоремы о редукции многомерной тэта-функции, накрывающей эллиптическую, и формул связи эллиптических функций Вейерштрасса с одномерными тэта-функциями также следует, что $\left(\dot{x}_{j} \equiv \partial_{y} x_{j}(y, z)\right)$

$$
w(x, y, z)=-\frac{3}{2} \sum_{j=1}^{n} \dot{x}_{j} \wp\left(x-x_{j}\right)+\text { const }_{1}, \quad \sum_{j=1}^{n} \ddot{x}_{j}=0
$$

ЗАМЕЧАнИЕ $6[1,2]$. Полюсы $x_{j}(y)$ функций $u(x, y)$ и $w(x, y)$ удовлетворяют динамике интегрируемой системы частиц Калоджеро-Мозера

$$
\ddot{x}_{j}=4 \sum_{k \neq j} \wp^{\prime}\left(x_{j}-x_{k}\right) \text {. }
$$

ЗАМЕЧАНИЕ 7 . Постоянная $c_{1}$ в формулах (1.2), (1.4а), (2.8) зависит от выбора базиса циклов на $Г$. В частности, если при автоморфизме $\tau(2.4) a$-циклы переходят в $a$-циклы, то $c_{1}=0$. 


\section{3. ПРИМЕР 1. ДВУХЗОННОЕ РЕШЕНИЕ}

Самое простое двухзонное эллиптическое решение уравнения Буссинеска может быть построено по четырехлистному накрытию $\Gamma_{2}^{4}$ :

$$
k^{4}-6 \wp(\alpha) k^{2}+4\left(\wp^{\prime}(\alpha)+h\right) k-3 \wp^{2}(\alpha)=0,
$$

где $h$ - вспомогательный параметр, $g_{2}=0, g_{3}=-h^{2}$.

Уравнение

$$
\chi^{3}=(E-5 h)^{2}\left(E^{2}+10 h E-83 h^{2}\right)
$$

описывает РП $\Gamma_{2}^{4}$ как трехлистное накрытие над сферой.

Связь между различными представлениями $Р П \quad \Gamma_{2}^{4}$ осушествляется с помошью соотношений

$$
\begin{aligned}
\wp(\alpha) & =\frac{(E-5 h)\left(E^{2}+10 h E-83 h^{2}\right)(E-13 h)}{16 \chi(E-4 h)^{2}}, \\
\wp^{\prime}(\alpha) & =\frac{\left(E^{2}+10 h E-83 h^{2}\right)^{2}}{32(E-4 h)^{3}}-h=\frac{(E-5 h)(E-13 h)^{3}}{32(E-4 h)^{3}}+h, \\
E & =\frac{\left(k^{3}-3 \wp(\alpha) k+\wp^{\prime}(\alpha)+h\right)^{2}}{2\left(\wp^{\prime}(\alpha)+h\right)}+4 h, \quad k=-\frac{3 \chi}{4(E-4 h)} .
\end{aligned}
$$

Накрытие (2.1), (3.1) соответствует редукции ненормированного голоморфного дифоференциала

$$
\sigma^{*} d \alpha=\frac{4 d E}{3 \chi}
$$

и ненормированного абелева дифференциала второго рода с единственным полюсом в точке $\mathcal{P}_{\infty}$

$$
d \Omega_{1}=\wp(\alpha) d \alpha-d k=\frac{(E-h)(E-5 h) d E}{3 \chi^{2}} .
$$

Поскольку поверхность $\Gamma_{2}^{4}$ имеет род $g=2$, она является гиперэллиптической и, следовательно, может быть представлена в виде двулистного накрытия сферы $\Gamma_{2}^{4}=$ $\{(\mu, \lambda)\}$ :

$$
\begin{gathered}
\mu^{2}=\lambda^{6}-40 h \lambda^{3}+432 h^{2}, \\
\mu=\frac{E^{2}-10 h E+33 h^{2}}{E-5 h}, \quad \lambda=\frac{\chi}{E-5 h}, \\
\frac{\lambda d \lambda}{\mu}=\frac{d E}{3 \chi}, \quad \frac{d \lambda}{\mu}=\frac{(E-5 h) d E}{3 \chi^{2}} .
\end{gathered}
$$

Легко видеть, что на $\Gamma_{2}^{4}$ существует мероморфная функция $\chi(P)$ с единственным полюсом четвертого порядка в точке $\mathcal{P}_{\infty}$ вида

$$
\chi=\xi^{-4}+O\left(\xi^{2}\right),
$$

и, следовательно,

$$
\Omega_{4}(P)=\chi(P), \quad V_{3}=0
$$

2 Теоретическая и математическая физика, т. 109, № 3, 1996 г. 
функции $u(x, y)$ и $w(x, y)$ не зависят от параметра $z$ и удовлетворяют "стационарным" высшим уравнениям КП (см. (1.6))

$$
\left\{\begin{array}{l}
w_{x x x}=3(w u)_{x} \\
w w_{x}=\frac{9}{8} u_{x} u_{x x}+\frac{9}{16} u u_{x x x}-\frac{9}{8} u^{2} u_{x}-\frac{1}{16} u_{x x x x x}
\end{array}\right.
$$

а $\psi$-функция является собственной функцией оператора четвертого порядка с эллиптическими потенциалами

$$
\partial_{x}^{4} \psi-2 u \partial_{x}^{2} \psi-\left(2 u_{x}+\frac{4}{3} w\right) \partial_{x} \psi-\left(\frac{5}{6} u_{x x}-\frac{1}{2} u^{2}+\frac{2}{3} w_{x}\right) \psi=\chi \psi .
$$

\section{4. ПРИМЕР 2. ТРЕХЗОННОЕ РЕШЕНИЕ}

Накрытие $\Gamma_{3}^{5}=\{(k, \alpha)\}$ определяется уравнением

$$
\begin{aligned}
k^{5}-10 \wp(\alpha) k^{3}+10\left(\wp^{\prime}(\alpha)+h\right) k^{2}- \\
-15 \wp^{2}(\alpha) k+2 \wp(\alpha)\left(\wp^{\prime}(\alpha)-5 h\right)=0,
\end{aligned}
$$

где $h$-вспомогательный параметр, $g_{2}=0, g_{3}=-25 h^{2}$.

Эта поверхность имеет род $g=3$ и может быть записана в виде

$$
\chi^{3}=(E-35 h)^{2}(E+45 h)\left(E^{2}+25 h E-1700 h^{2}\right) .
$$

Связь между различными представлениями РП $\Gamma_{3}^{5}$ осушествляется с помошью соотношений

$$
\begin{aligned}
\wp^{\prime}(\alpha) & =\frac{2(E-115 h)^{3}\left(E^{2}+25 h E-1700 h^{2}\right)}{125(E-19 h)^{3}(E+45 h)}+5 h= \\
& =\frac{2(E+125 h)^{3}(E-35 h)^{2}}{125(E-19 h)^{3}(E+45 h)}-5 h, \\
\wp(\alpha) & =\frac{(E-115 h)(E+125 h) \chi}{25(E-19 h)^{2}(E+45 h)}, \quad E=-\frac{4}{5 k} \partial_{k} \mathcal{R}(k, \alpha)-29 h, \\
k & =-\frac{4(E-35 h)\left(E^{2}+25 h E-1700 h^{2}\right)}{5(E-19 h) \chi} .
\end{aligned}
$$

Накрытие (2.1), (4.1) соответствует редукции ненормированного голоморфиного дифиференциала

$$
\sigma^{*} d \alpha=\frac{5(E+29 h)(E-35 h) d E}{3 \chi^{2}}
$$

и ненормированного абелева дифференциала второго рода с единственным полюсом в точке $\mathcal{P}_{\infty}$

$$
d \Omega_{1}=\wp(\alpha) d \alpha-d k=\frac{(E-15 h) d E}{3 \chi} .
$$


Поскольку $\chi(P)$ - мероморфная функция с единственным полюсом пятого порядка в точке $\mathcal{P}_{\infty}$ вида

$$
\chi=\xi^{-5}+O(\xi)
$$

Tо

$$
\Omega_{5}(P)=\chi(P), \quad V_{4}=0
$$

функции $u(x, y)$ и $w(x, y)$ удовлетворяют “стационарным” уравнениям КП более высокого порядка, чем (3.5), а $\psi$-функция является собственной функцией оператора пятого порядка с эллиптическими потенциалами.

Автор благодарит В.Б.Матвеева, М.А.Салля и В.З.Энольского за полезные обсуждения.

Работа выполнена при финансовой поддержке Российского фонда фундаментальных исследований (95-01-00686).

\section{Список литературы}

[1] H. Airault, H.P. McKean, J. Moser. Comm. Pure Appl. Math. 1977. V. 30. P. 95-148.

[2] И.М. Кричевер. Функц. анализ и его прилож. 1980. Т. 14. Вып. 4. С. 45-54.

[3] А.Р. Итс, В.З. Энольский. Функц. анализ и его прилож. 1986. Т. 20. Вып. 1. С. 73-74.

[4] Б.А. Дубровин, С.П. Новиков. ЖЭТФ. 1974. Т. 67. Вып. 12. С. 2131-2143.

[5] В.Е. Захаров, С.В. Манаков, С.П. Новиков, Л.П. Питаевский. Теория солитонов: метод обратной задачи. М.: Наука, 1980.

[6] Е.Д. Белоколос, А.И. Бобенко, В.Б. Матвеев, В.З. Энольский. УМН. 1986. Т. 41. Вып. 2. С. 3-42.

[7] J.-L. Verdier. In: Algebraic Analisys. Vol. II (Micio Sato Sixtieth Birthday Vol.). Academic Press, 1988. P. 901-910.

[8] A. Treibich. Duke Math. J. 1989. V. 59. № 3. P. 611-627.

[9] A. Treibich, J.-L. Verdier. Solitons elliptiques. Volume en l'honneur du $60^{e}$ anniversaire du prof. A.Grothendieck. Boston: Birkhäuser, 1990.

[10] Е.Д. Белоколос, В.З. Энольский. УМН. 1989. Т. 44. Вып. 5. С. 155-156.

[11] Е.Д. Белоколос, В.З. Энольский. Функц. анализ и его прилож. 1989. Т. 23. Вып. 1. С. 57-58.

[12] А.О. Смирнов. Мат. заметки. 1989. Т. 45. Вып. 6. С. 66-73.

[13] E.D. Belokolos, A.I. Bobenko, V.Z. Enol'skii, A.R. Its, V.B. Matveev. Algebro-geometrical approach to nonlinear evolution equations. Springer Ser. Nonlinear Dynamics. Berlin-Heidelberg-New York: Springer, 1994.

[14] A.O. Smirnov. Acta Appl. Math. 1994. V. 36. P. 125-166.

[15] V.Z. Enol'skii, N.A. Kostov. Acta Appl. Math. 1994. V. 36. P. 57-86.

[16] E.D. Belokolos, V.Z. Enol'skii. Acta Appl. Math. 1994. V. 36. P. 87-117.

[17] А.И. Бобенко. Функц. анализ и его прилож. 1984. Т. 18. Вып. 3. С. 74-75.

[18] А.О. Смирнов. Мат. заметки. 1989. Т. 46. Вып. 5. С. 100-102.

[19] А.О. Смирнов. Мат. сборник. 1990. Т. 181. Вып. 6. С. 804-812.

[20] А.О. Смирнов. ТМФ. 1994. Т. 100. № 2. С. 183-198.

[21] А.О. Смирнов. Мат. сборник. 1994. Т. 185. Вып. 8. С. 103-114.

[22] А.О. Смирнов. Мат. заметки. 1995. Т. 58. Вып. 1. С. 86-97.

[23] А.О. Смирнов. Мат. сборник. 1995. Т. 186. Вып. 8. С. 134-141.

[24] В.Б. Матвеев, А.О. Смирнов. ДАН СССР. 1987. Т. 293. Вып. 1. С. 78-82.

[25] В.Б. Матвеев, А.О. Смирнов. В кн.: Распространение волн. Теория рассеяния (Проблемы математической физики, вып. 12). Л.: ЛГУ, 1987. С. 225-236.

[26] V.B. Matveev, A.O. Smirnov. Lett. in Math. Phys. 1987. V. 14. P. 25-31.

[27] G.H. Halphen. Mem. pres. l'Acad. de Sci. de France. 1884. V. 28. P. 1-300.

[28] Э. Камке. Справочник по обыкновенным дифференциальным уравнениям. М.: Физматгиз, 1961. 
[29] V.P. Gerdt, N.A. Kostov. In: Computers and Mathematics. Eds E.Kaltofen, S.M.Watt. Berlin-Heidelberg: Springer, 1989. P. 277-283.

[30] J.C. Eilbeck, V.Z. Enolskii. J. Math. Phys. 1994. V. 35. № 3. P. 1192-1201.

[31] Yu.V. Brezhnev. Phys. Lett. A. 1996. V. 211. P. 94-100.

[32] И.М. Кричевер. ДАН СССР. 1976. Т. 227. Вып. 2. С. 291-294.

[33] И.М. Кричевер. УМН. 1977. Т. 32. Вып. 6. С. 180-208.

[34] Э.И. Зверович. УМН. 1971. Т. 26. Вып. 1. С. 113-179.

[35] V.B. Matveev. Abelian functions and solitons: Preprint № 373. Univ. of Wrocław, 1976.

[36] A. Hurwitz. Math. Ann. 1893. V. 41. P. 403-442.

[37] F.K. Schmidt. Math. Zeit. 1938. V. 45. P. 75-96.

[38] В.В. Сильвестров, Л.И. Чибрикова. Изв. вузов. Математика. 1979. № 12. С. 51-56.

[39] Н.И. Ахиезер. Элементы теории эллиптических функций. М.: Наука, 1970.

Поступила в редакцию 17.IV.1996 г.

\section{A. O. Smirnov \\ ON SOME SET OF ELLIPTIC SOLUTIONS OF THE BOUSSINESQ EQUATION}

An ansatz concerning Krichever curves is considered for two-periodic finite-gap solutions of the Boussinesq equation. These solutions are expressed in terms of equianharmonic elliptic Weierstrass $\wp$-functions. Examples are given. 